\title{
Oxygen Delivery on Medical Wards
}

\author{
Christopher Dickson
}

South Devon Healthcare NHS Foundation Trust

\begin{abstract}
Oxygen is used widely used on all medical wards. It is a drug and should be prescribed. There are known problems with over and under delivery of oxygen to patients. Through national audits and recording baseline data locally, compliance with prescribing is low. This has potentially serious patient safety issues. This quality improvement project attempted to improve oxygen prescribing and subsequent dose adjusting on various medical wards. Monitoring showed a transient improvement but this was not sustained. As a result of this project further research will be put into developing the electronic observation chart to set parameters for target saturations.
\end{abstract}

\section{Problem}

Oxygen is sporadically prescribed and clear instructions to nursing staff in regard to target saturations is insufficiently communicated and documented. The weaning process is also not routinely monitored and done on an ad-hoc basis. This results in either patients being on too much oxygen or on oxygen for too long a period. This can result in a longer admission as there is often a delay in recognising and instigating a change in oxygen supply.

\section{Background}

The British Thoracic Society published guidelines in 2008 stating that oxygen should be treated as a drug and should be prescribed. Emergency oxygen delivery has been audited nationally since then and although there has been some improvement, delivery of oxygen remains a problem nationally. Significant complications can arise if too much or too little oxygen is given and if oxygen delivery is longer than is clinically indicated it prolongs weaning time. This can be a factor which delays discharge from hospital.

The process flow of oxygen delivery in our trust identified the key point in the process was the nursing decision to increase or decrease oxygen. The other key point was doctors prescribing oxygen initially. The main focus of the quality improvement project was to improve the initial identification of people requiring oxygen and doctors highlighting this to nurses by prescribing it. The second will be to improve the decision making on how much oxygen to give patients.

See supplementary file: ds4945.pdf - "Process Flow"

\section{Baseline measurement}

This project focused on the amount of patients on oxygen with this prescribed. This would be recorded by auditing all patients, on two medical wards, who were having oxygen as documented on the observation charts.

\section{Design}

The drug chart used in the hospital already provides a pre-printed section for oxygen prescribing. The use of this section is somewhat erratic and varies between wards. Using the section already printed allows for easy implementation. It was decided that talking to doctors directly would result in greater increase in compliance with filling in the section on the drug chart. Although this is time consuming if this was successful then using other methods to disseminate this would be easier if it was demonstrated to be working on the trial wards.

\section{Strategy}

PDSA Cycle 1- A single bay on one elderly care ward was chosen to attempt to implement a process of reviewing saturations and reducing the oxygen accordingly. This, however, was not successful and did not result in reviewing of oxygen saturations and effort to wean oxygen.

PDSA Cycle 2- The first cycle proved to be too complicated to implement in a single step. We then reverted to ensuring that all the doctors on two wards were prescribing oxygen ( 1 mixed medical ward and the respiratory ward). Although we managed to improve oxygen prescribing this did not result in a corresponding review of oxygen in relation to saturations. This was mainly because the recording of observations was done separately to the drug rounds and often done by different people.

PDSA Cycle 3- On achieving improved prescribing it became clear that the drug chart was not looked at sufficiently. The oxygen saturations and respiratory rates were recorded routinely on the electronic observation charts (VitalPAC). Currently this does not allow for changing of target saturations and all patients score if saturations are less then $94 \%$. Further investigation will be made to see if the VitalPAC will allow for custom target saturations and escalate the early warning score if outside these parameters.

See supplementary file: ds4943.pdf - "PDSA 1" 


\section{BMJ Quality Improvement Reports}

\section{Post-measurement}

Patients on oxygen were reviewed weekly to see if they had oxygen prescribed. A run chart was plotted to record this information. The baseline showed that approximately $30 \%$ on the respiratory ward and about $10 \%$ on the general medical ward on oxygen had it prescribed.

Following the second PDSA cycle this transiently improved prescribing but then returned to baseline.

See supplementary file: ds4947.pdf - "Results"

\section{Lessons and limitations}

The main thing I learnt from this project is the involved stakeholders in this project are already very busy and any change that requires more input must demonstrate an improvement. If stakeholders do not feel the change results in improvement it is difficult to sustain change.

What became clear after the first 2 PDSA cycles was that creating a perceived increase in work results in limited improvement. I found that having the observation chart and oxygen prescription chart separate resulted in duplicate recording of saturations. This resulted in nurses neglecting the prescription chart and subsequently doctors started to fail to prescribe at a result of this. What I will work on following this project will be try to change a task that is already done and result in a process that forces action by the user. With implementation into the electronic observation chart this should help to address this.

The BTS guidelines were implemented in 2008 and are regularly audited, this demonstrates that the audit model of improvement has had little success in driving change so far. The attempt to try and make oxygen regarded as a drug that should be prescribed still has not been widely adopted. The perception that oxygen is relatively harmless still prevails. This is exacerbated by warning scores that give a lower score despite the specific patient potentially being outside their target range of saturations. This further needs to be addressed by education.

\section{Conclusion}

This project has highlighted that we are struggling to meet the guidelines set out by the BTS on oxygen prescribing. The benefits of adhering to these are twofold. Firstly, it is important for patient safety to ensure patients have the appropriate oxygen delivered to them. Secondly, more diligent use of oxygen should result in a shorter time patients are on oxygen and in theory reduce the length of stay for patients. The current system is failing because of the lack of linking the observation of saturations to the thought to reduce oxygen supply. The main factor involved with this is the general perception that if saturations are high then no further action is required.

Use of electronic observation charts should allow the use patient specific saturation targets which will also then allow for escalation of their early warning score and hopefully prompt action. This will be explored and if feasible will hopefully result in a greater improvement. This should result in a direct response as if someone's early warning score is increased when saturations are outside the target range, this is difficult to ignore.

\section{References}

1. BR O'Driscoll, LS Howard, AG Davison. Guideline for emergency oxygen use in adult patients. Thorax Oct 2008 Vol 63 Suppl VI.

2. BR O'Driscoll, et al. Audit update, British Thoracic Society emergency oxygen audits. Thorax Apr 2011 (Online) 10.1136/thoraxjnl-2011-200078.

\section{Declaration of interests}

Nothing to declare

\section{Acknowledgements}

Project Supervisor- Dr G Kendall

Assistance with project- Dr S Craig 